\title{
EXPEDITION DE POISSONS VIVANTS DE SOLOGNE AUX HALLES DE PARIS
}

\author{
Par M. Robert de BALLOY
}

Pisciculteur aux Maremberts (Loir-et-Cher).

\section{I. - Distance kilométrique : I8o kilomètres.}

II. - Moyen de transport : Camionnette bàchée.

L'enduit de la bâche ne doit pas être nocif pour le Poisson. Une bâche verte, mise à tremper dans un bassin contenant des Poissons témoins, les a fait crever. Elle a été changée contre une bâche blanche qui a donné satisfaction.

Dimensions de la bâche : 5 mètres sur 6 .

Prix environ 600 francs.

Primitivement, je ne trouvais sur place que des camionnettes "Citroën " r.ooo kilos; ce qui, pour une voiture de location, met le prix du voyage trop cher. Si la camionnette appartenait au propriétaire, elle pourrait suffire.

J'emploie maintenant des "Ford ", 2 tonnes, que je loue aux entrepreseurs de tranport.

\section{III. - Personnel employé.}

Un homme suffirait, mais il vaut mieux faire accompagner le chauffeur par un préposé du propriétaire pour que le camion ne soit pas abandonné pendant les formalités de l'octroi, ou pour remettre de l'eau en cours de route, et surtout pour vérifier à l'arrivée la proportion de Carpess mortes.

IV. - Heures et durée du transporl.

Théoriquement, les Halles sont ouvertes aux envois de cette sorte entre 9 et to heures du matin. Il faut compter 5 à 6 heures de transport, soit le départ à 3 ou 4 heures du matin. En réalité, cette heure-là est plus commode pour le mandataire, les forts de la Halle sont alors à sa disposition.

Maintenant je procède ainsi : le mandataire est prévenu de l'heure d'arrivée du camion. (Un coup de téléphone vers 9 heures, quand la pêche est à moitié faite, et que je vois à quelle heure le camion pourra partir). Il se débrouille pour avoir du monde sous la main à l'arrivée. G'est une simple question de pourboire.

\section{V. - Méthode.}

Le jour de la pêche, je trie les Carpes de I kilogramme et plus, pesant 26 kilos fort pour 25 kilos. Je les mets dans des cageots à gibier à lattes rupprochées, ficelés, qu'une voiture emporte à mesure. Je les mets à flotter dans un étang dont la chausséc est praticable au camion. Les Carpes se 
nettoient et reprennent de la vigueur. Si l'on avait de l'eau courante, ce serait encore mieux.

Quand la pêche est finie et le Poisson reposé, je charge le camion.

J'ai eu soin d'abreuver la bâche pour qu'elle garde l'eau. Il est difficile de dire quelle quantité d'eau est nécessaire : les camions "Ford " ont des ressorts très complaisants. Je mets de l'eau jusqu'à ce que les Poissons puissent nager sans se gêner, et encore un peu. Plus la plate-forme est rrande, mieux cela vaut. Je n'ai jamais remis d'eau en route ; mais il pourrait être utile de faire suivre des seaux en toile et de compléter en cours de route avec de l'eau de pluie, si l'on voyait le Poisson souffríi. Ie n'ai pas essayé l'oxygène.

Jusqu'ici, je n'ai pas chargé suffisamment les camions. Je crois qu'on

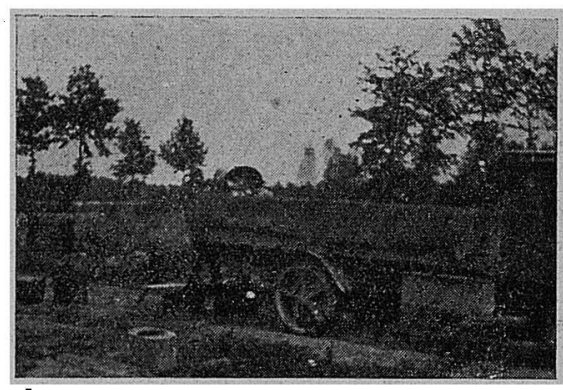

Fig. 21. - Chargement d'un camion à l'étang. peut, sans danger, mettre 700 kilogs de Carpes et environ deux fois leur poids d'eau. Le plus important est de ne pas expédier du Poisson fatigué.

\section{VI. - Mortalité.}

J'ai eu plus de mortálité avec l'ancien sytème : départ à 3 heures du matin. Les Poissons, en cageots depuis la veille étaient fátigués. J'ai même vu crever les trois quarts d'un envoi avant le départ. Le temps s'était hrusquement mis à l'orage dans la nuit, et les cageots étaitent trop rapprọchés devant la bonde d'un petit étang.

Ii faut toujours compter sur to ̀े $15 \%$ de Poissons vendus morts.

VII. - Epoque des envois.

Il y a une grosse demande en Septembre, au moment des fêtes juives. (Se renseigner sur la date exacte). J'ai, plusieurs fois, manqué les meilleurs r.rix, ne pouvant être prêt à temps. Le reste de l'année, on ne peut guère obtenir plus de 8 francs à $8 \mathrm{fr}$. 50 le kilog, soit 5 francs à $5 \mathrm{fr}$. 50 net, en comptant les ennuis pour rien. Pendant quelques années, les cours ont été élevés fin Avril et Mai. En plus de la difficulté de pêcher à cette date, on risque de perdre beaucoup de Poisson. Cette année-ci, fin Juin, les cours n' se sont pas encore relevés.

Il faut, autant que possible, envoyer des lots homogènes. Pas de Carpe de moins d'un kilogramme. Un lot important de Carpes plus grosses serait vayé très cher par les Juifs.

J'ai vendu $\mathbf{2}$ francs le kilo, et j'ai vu les cours à I4 francs. Il y a un facteur chance qui intervient, et il faut faire plusieurs envois pour avoir une moyenne élevée.

VIII. - Frais.

Le camion de l'entrepreneur coûte 3 francs du kilomètre. Pour plusieurs 
voyages, je peux obtenir 2 fr. 5o. L'octroi, 22 francs aux roo kilos (1). La commission du mandataire, $8 \%$.

Frais d'abri variables, probablement avec le temps que le Poisson est entreposé avant la vente.

J'ai toujours compté 3 francs de frais par kilogramme. Avec camionnette r.ooo kilogs ( 1 fr. 50 du kilomètre); en camion de 2 tonnes ( 3 francs du kilomètre). En chargeant davantage, on devrait descendre à 2 fr. 50 .

IX. - Détail des envois.

5 Octobre r 929 . - Départ à 3 hcures du matin.

Carpes vivantes, 260 kilos ......... 2.36r fr. "

— mortes, 4i kilos .......... 205 " $2.566 \mathrm{fr}$. "

I I Octobre rg29. - Départ à 3 heures du matin.

Carpes vivantes, 240 kilos ........ 3. r 20 fr. "

- mortes, 50 kilos ............ 350 "

Tanches vivantes, IOI kilos ......... r.o6o "

- mortes, rg kilos $\ldots \ldots \ldots \ldots \ldots, 9^{5}$

(Ces Tanches ont voyagé à sec, en cageot, sur le porte-bagages).

Frais pour les 2 envois :

Abri ................... $\delta_{\text {I fr. " }}$

Commission .............. $5_{7} 5$ 3o

Octroi (environ) $\ldots \ldots \ldots \ldots \ldots \ldots \ldots \ldots \ldots, 140$,

2 voyages à 600 francs $\ldots \ldots \ldots \ldots \ldots$ I. $200 \quad$ r. 996 fr. 30

Net ..... 5.r 95 fr. 20

Prix de vente net pour Carpes et Tanches : 7 fr. 3o.le kilogramme.

$\mathrm{I}^{\text {er }}$ Octobre I 930 . - Départ à midi, après la pêche, par grosse chaleur.

Carpes vivantes, $483 \mathrm{~kg} 700 \ldots \ldots \ldots 4.857 \quad 85$

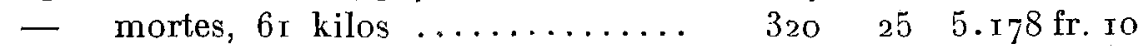

Frais :

Abri

$64^{\circ} 20$

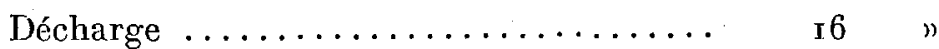

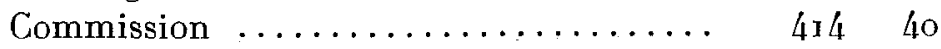

Octroi .................. 118 80

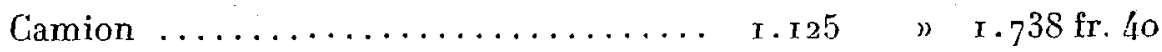

Net $\ldots \ldots, 3.439 \mathrm{fr} .7 \mathrm{o}$

Prix de vente au kilogramme : brut, 9 fr. 50 ; net, 6 fr. 35 :

(r) Chiffre à vérifier. Je me suis basé sur le prix payé par moi, mais il y a peut-être uṇe méthode de calcul plus compliquée que lạ division. 


\section{X. - Expédition de Poissons morts.}

Un des avantages de l'expédition du Poisson aux Halles, c'est que l'on dispose de la totalité de sa pèche et que, proportionnellement, on fait un plus grand bénéfice sur le reste de la pêche que sur la Carpe.

Les frais de transport ne sont pas ce que l'imaginent les gens qui ne se servent du chemin de fer que pour de petites expéditions.

Voici ceux de I929, qui n'ont pas dû beaucoup varier : 21 I fr. 85 la tonne avec minimum de 5 o kilogs.

Ainsi, j'ai vendu, en rg3o-3r, le poids net de r.262 kilos de Poisson ct j'ai déboursé au Chemin de fer la somme de $306 \mathrm{fr}$. 50 (I).

En Mars r 929, 25o kilos de Brochets ont été vendus par un de mes voisins $I_{7}$ francs le kilo brut, soit $\mathrm{I} / 4 \mathrm{fr}$. 50 net.

Le 5 Octobre 1929 , 3o kil. 200 Brochets, 546 fr. го.

I $-\frac{50 \text { kil. }}{80 \text { kil. } 200}-\frac{900 \mathrm{fr} . " \prime}{\text { I.446 fr. ro. }}$

Moyenne, brut is francs le kilog.

Ces derniers envois ayant été effectués par la camionnette transportant des Carpes vivantes, il est difficile de leur attribuer une part des frais de transport. Il y aurait lieu de déduire la commision de $8 \%$. Les frais de toute nature s'élèvent à $15 \%$.

J'ignore si les Halles absorberaient de grandes quantités de friture ; en tout cas, les prix nets obtenus ont toujours été supérieurs aux cours locaux.

\section{CONSULTATIONS TEGHNIQUES}

4) D. - Qu'est-ce que le coefficient de filtration dont il est question dans l'article de M. le professeur Andé́, sur l'empoissonnement des lacs de bartage ? (2) (U. P., à Paris).

4) R. - Lorsqu'on effectue des pêches de plancton, au moment de la remontée du filet, une certaine quantité d'eau est rejetée en dehors et ne filtre pas. Le volume de plancton obtenu est donc inférieur à celui qui eût élé recueilli si toute la colomne d'eau se trowvant au-dessus du flet eût passé par les mailles. On corrige cette erreur en multipliant le volume du plancton récolté par le coefficient de filtration. Celui-ci est obtenu en faisant passer effectivement par le filet la quantité d'eau qui, théoriquement, devrait y passer, et en dosant ensuite le plancton. Pour cela, on puise l'eau à la surface dans un grand récipient et on la verse dans le filet ; il est préfórable, pour diverses raisons, de ne pas puiser cette eau avec la pompe à plancton.

Le coefficient ne sera que largement approximatif, car il varie suivant l'âge du filet, ia nature du plancton, etr.

Nora. - Aux termes de l'article III du règlement du Bulletin, les réponses données aux questions posées le sont sans garantie de l'Union piscicole de Trance on du Comité de Rédaction.

(1) Voir les détails pour la verite de la friture au Bulletin de Juin $\mathbf{r g}^{3} \mathbf{1}_{\text {; }}$ page 386.

(2) Voir Bulletin, Juin $193 \mathrm{~J}$, p. 362 : 